Leading Article

\title{
The spectrum of schizophrenia
}

\author{
Malcolm P.I. Weller
}

Friern Hospital, Friern Barnet Road, London N11 3BP, UK.

Schizophrenia is a subset of psychosis but includes more besides, and this additional domain excites contention; a domain that has expanded and shrunk according to prevailing preferences. The subtle boundaries of this non-psychotic domain are important in many practical ways, including forensic and community psychiatric care issues. ${ }^{1,2}$ The subset of psychosis is easier to define and most diagnostic schemes concur on this, universally according Schneider's symptoms of the first rank a central place ${ }^{3}$ (although clinical skill has to be exercised in probing for psychotic phenomena and assessing the responses, such as differentiating between hallucinations and pseudo-hallucinations). Disagreements rest, in part, on how long these psychotic symptoms should have been present for the diagnosis to be certain.

There is hesitancy in describing brief lived psychoses as schizophrenia, and the term thereby comes to denote a chronic disorder. Such brief lived illnesses have been variously termed schizophreniform, cycloid psychosis, boufée delirante, and a number of syndromes allegedly limited to specific cultures, such as amok $^{4}$ and spirit possession or boufée delirante aigue. ${ }^{5}$ Despite the implication that these terms describe transient states different from schizophrenia, these conditions tend to reappear as indubitable schizophrenia and to be found in family members of schizophrenic probands.

The prodromal symptoms of schizophrenia are likely to be difficult to recognize for what they are. Kraepelin ${ }^{6}$ described affective and neurotic symptoms as sometimes representing manifestations, whilst 'The general trend of volition and also the higher emotions might form the first point of attack.' Bleuler ${ }^{7}$ described a related situation; 'Where only the basic symptoms are visible, we speak of schizophrenia simplex (the primary dementia of earlier authors). It is usually a case of a dementia in the sense of schizophrenia that

Correspondence: M.P.I. Weller, M.A., M.B., B.S., F.R.C. Psych., F.B.Ps.S.

Received: 15 July 1987. increases gradually in the course of decades. The anamnesis invariably indicates that the disease has been mistaken for years; it was a latent schizophrenia.' This concept of latent schizophrenia has been ridiculed as nonsensical and the ability to recognize such a state has been doubted, but there are many who feel they have seen similar situations to those that Bleuler described in characteristic oddities of personality and, whilst hesitating to be emphatic, often believe that they are dealing with a case of evolving schizophrenia in which the symptoms have not yet crystallized sufficiently to justify the term. The implications of diagnosing schizophrenia are such that there will be considerable hesitancy in voicing suspicions that one may be witnessing such a developing situation, particularly in young people who are inevitably passing through periods of difficult adjustment, yet who represent the group in whom the onset is particularly likely.

Hoch \& Polatin ${ }^{8}$ emphasized that neurotic symptoms are common in evolving schizophrenia in Western culture. The possibility of later schizophrenia in neurotic conditions had been noted by Jung in his correspondence with Freud,' 'I have often had cases that passed with apparent smoothness from hysteria or obsessional neurosis straight into D. pr.' (dementia praecox), and the same relationship is emphasized in follow-up studies. ${ }^{10-12}$

Some researchers claim an overlap between borderline and schizotypal personality disorder, with common features to the characteristic premorbid disposition of schizophrenics, and others argue for separate disorders with differing outcomes. ${ }^{13,14}$ In the most recent third edition of the American Diagnostic and Statistical Manual (DSM-III) the two terms are defined separately, schizotypal personality disorder being characterized by suspicious, paranoid attitudes, sensitivity to criticism, ideas of reference, poor rapport, and odd ways of communication. The DSM-III criteria for borderline personality disorder, in its typical unambiguous but unweighted way, that has been uncharitably referred to as the Chinese menu 
approach, require at least four of the following impulsiveness, self-damaging acts, unstable interpersonal relationships, intolerance of being alone, empty and bored and identity disturbance, the last seeming more akin to schizophrenia and the first to psychopathy.

\section{Genetics}

Bleuler ${ }^{7}$ remarked on a number of peculiarities in the relatives of his schizophrenic patients, which stopped short of frank schizophrenia but seemed to represent minor features of the disease. Kraepelin ${ }^{6}$ similarly observed that 'not infrequently one learns further that among the brothers and sisters of the patient there are found striking personalities, criminals, queer individuals, prostitutes, suicides, vagrants, wrecked and ruined human beings, all being forms in which more or less well-developed dementia praecox may appear.' (p234) Similar observations have continued to be made, with an excess of borderline conditions in the first degree relatives, whilst some relatives have 'clearly schizoid or paranoid personalities as well as others characterized by schizoid features and serious emotional disorders . . .15

Kendler et al. ${ }^{16}$ confirmed the higher incidence of schizotypal personality disorder in the biological relatives of adopted-away schizophrenics found by Kety et al. ${ }^{17}$ using a methodology that met criticisms of earlier studies ${ }^{18}$ but pointed out that there is some circularity in the arguments, in that the DSM-III concept of schizotypal personality derived from an examination of the case summaries of the biological relatives of the adopted-away schizophrenics in the extended family study of Kety et al. ${ }^{17}$

These same personality deficiencies are found as prodromal features of later schizophrenia. Psychopathic features also occur, emphasizing a relationship between the two conditions, which have other biological features in common ${ }^{19}$ and psychopathy is over-represented in the families of schizophrenic probands. ${ }^{20,21}$ If these non-schizophrenic individuals with allegedly schizophrenic spectrum traits ${ }^{22}$ are included in statistical comparisons the genetic transmission of schizophrenia is more convincing. ${ }^{17,23,24}$

The relatives of schizophrenics are at greater risk than a random population of developing schizophrenia, the risk being proportional to the degree of consanguinity. Where a family history is absent sporadic cases of schizophrenia betray evidence of brain damage. ${ }^{25} \mathrm{~A}$ recent familial study, in which the probands were followed-up over a long period and in many cases personally interviewed by the researchers, showed that brief lived schizophreniform psychoses, schizoaffective psychoses and psychotic affective illness betrayed a schizophrenic pattern of inheritance. ${ }^{26}$

\section{Organic findings}

In view of these genetic findings it is of great interest that, in addition to certain personality factors which are allegedly detected in the apparently unaffected first degree relatives, claims have been made that they betray cognitive dysfunction and formal thought disorder on objective tests. ${ }^{27,28}$

Neurological abnormalities similar to those found in schizophrenics have also been found in their, apparently, unaffected first degree relatives, initially found as eye tracking dysfunction ${ }^{29-31}$ but more recently apparent in a battery of conventional neurological tests $^{32}$ suggesting that the neurological signs in schizophrenia are unlikely to be entirely consequential on current or past medication. The extent of the dysfunction is modestly, but significantly, associated with the degree of thought disorder. ${ }^{31}$

\section{Treatment}

Neuroleptic treatment of schizotypal personality disorder produces a perceptible improvement, but is poorly tolerated ${ }^{33}$ and also produces modest improvements in borderline disorders, ${ }^{34,35}$ as evaluated in a double blind trials. ${ }^{36,37}$ The improvement produced by neuroleptics typically includes improvement in depression and anxiety, and Goldberg et al..$^{38}$ alsơ found improvement in obsessive-compulsive problems, suggesting that these are components of the total complex, rather than independent features, particularly since the tricyclic antidepressant, amitriptyline, seemed to produce a deterioration in some patients.

\section{Conclusion}

As far as we can tell it would seem that the concept implicit in the words of Lewis \& Piotrowski ${ }^{39}$ that 'a little schizophrenia is schizophrenia' can be extended to a little schizophrenia-like phenomena might well be schizophrenia, whether considering inheritance, brief lived psychotic episodes, or personality characteristics.

The organic findings in the spectrum disorders, and even in the apparently unaffected blood relatives, imply that these disorders are medical illnesses. The possibility that low doses of antipsychotic medication may be helpful should be kept in mind and such an approach can prove very rewarding; a vivid demonstration of this is described elsewhere. ${ }^{19}$

\section{Acknowledgements}

I thank Friern Hospital library for considerable help. 
1. Weller, B.G.A., Weller, M.P.I., Cocker, E. \& Mahomed, S. Crisis at Christmas 1986. Lancet 1987, i: 553-554.

2. Weller, M.P.I. Future psychiatric services: Planning for illness of distress? Nature, in press.

3. Weller, M.P.I. Some problems in the diagnosis of schizophrenia and the concept of clear consciousness. In Stevens, R. (ed) Aspects of Consciousness. Academic Press, London, New York, Tokyo, 1984, pp 117-166.

4. Kraeplin, E. Vergleichende psychiatrie (Translated as Comparative Psychiatry) In Hirsch, S.R. and Shepherd, M. (eds) Themes and Variations in European Psychiatry. Wright, Bristol, 1974, p 1904.

5. Sanseigne, A. \& Desrosiers, M. Evaluation of psychopharmaceuticals in an undeveloped country. In Kline, N.S. (ed) Psychiatry in the Underdeveloped Countries: Report of a Roundtable Meeting, APA Annual Meeting Atlantic City, 12 May, 1960 pp 52-8, cited Kiev 1972 p. 103.

6. Kraepelin, E. In Robertson, G.M. (ed) Demential Praecox Trans. M. Barclay. E. and S. Livingstone, Edinburgh, 1919. p76.

7. Bleuler, E. Textbook of Psychiatry, translated by A.A. Brill. George Allen and Unwin, London, 1923. p 432-3.

8. Hoch, P.H. \& Pollatin, P. Pseudoneurotic forms of schizophrenia. Psychiatr $Q$ 1949, 23: 248-276.

9. Jung, C.C. In Manheim, R. and Hull, R.F.C. (eds). The Freud/Jung Letters. Hogarth Press and Routledge and Kegan Paul, London and Henley-on-Thames, 1907, pp 35-38.

10. Carter, A.B. The prognosis of certain hysterical symptoms. Br Med J 1949; 1: 1076-1079.

11. Ljungberg, L. Hysteria: a clinical, prognostic and genetic study. Acta Psychiatrica Neurolgica Scandinavica 1957, 32: Suppl. No. 112.

12. Slater, E. The diagnosis of hysteria. $B r$ Med J 1965, 1: 1395-1399.

13. Torgersen, S. Genetics of neurosis: the effects of sampling upon the twin concordance ratio. Br J Psychiatry 1983, 142: 126-132.

14. Plakum, E.M., Burkhardt, P.E. \& Muller, J.P. Fourteen year follow-up of borderline and schizotypal personality disorders. Compr Psychiatry 1945, 26: 448-455.

15. Alanen, Y.O. In search of the international origin of schizophrenia. In Cancro, R., and Dean, S.R., Research in the Schizophrenic Disorders: The Stanley $R$. Dean Award Lectures, vol. 1. Spectrum Publications, Jamaica, 1985, p 253.

16. Kendler, K.S., Gruenberg, A.M. \& Strauss, J.S. An independent analysis of the Copenhagen sample of the Danish adoption study of schizophrenia: II The relationship between schizotypal personality disorder and schizophrenia. Arch Gen Psychiatry 1981, 38: 982-984.

17. Kety, S. Rosenthal, D., Wender, P. \& Schulsinger, F. Mental illness in biological and adoptive families of adoptive schizophrenics. Am J Psychiatry 1971, 128: $302-306$.

18. Liebowitz, M.R. Is borderline a distinct entity? Schizophrenia Bulletin 1979, 5: 23-38.

19. Weller, M.P.I. Medical concepts in psychopathy and violence. Med Sci Law 1986, 26 (2): 131-143.
20. Heston, L. Psychiatric disorder in foster home reared children of schizophrenic mothers. Br J Psychiatry 1966, 112: 819-825.

21. Stephens, D.A., Atkinson, M.W., Kay, D.W.K., Roth, M. \& Garside, R.F. Psychiatric morbidity in parents and sibs of schizophrenics and non schizophrenics. Br J Psychiatry 1975, 127: 97-108.

22. Inouye, E. Similarity and dissimilarity of schizophrenia in twins. In Proceedings of the Third World Congress of Psychiatry, Vol. 1. University of Toronto Press, Montreal, 1961, pp 524-530.

23. Kety, S., Rosenthal, D., Wender, P. \& Schulsinger, F. The type and prevalence of mental illness in the biological and adoptive families of adopted schizophrenics. In Rosenthal, D. and Kety, S. (eds) The Transmission of Schizophrenia. Pergamon, Oxford, 1968.

24. Wender, P., Rosenthal, D., Kety, S., Schulsinger, F. \& Weiner, J. Crossfostering research strategy for clarifying the role of genetic and experimental factors in the etiology of schizophrenia. Arch Gen Psychiatry 1974, 30: 121-128.

25. Reveley, A.M., Reveley, M.A. \& Murray, R.M. Cerebral ventricular enlargement in non-genetic schizophrenia: A controlled twin study. Br J Psychiatry 1984, 144: 89-93.

26. Kendler, K.S., Gruenberg, A. \& Tsuang, M.T. A DSM-III family study of nonschizophrenic psychotic disorders. Am J Psychiatry 1986, 143: 1098-1105.

27. Romney, D.M. Formal thought disorder amongst schizophrenics: a new look at some old data. J Clin Psychol 1984, 40: 51-52.

28. Callahan, L.A. \& Saccuzzo, D.P. Associative intrusions in the verbal behaviour of the first-degree relatives of adult schizophrenics. A preliminary study. J Nerv Ment Dis 1986, 174: 240-242.

29. Holzman, P.S., Proctor, L.R. \& Hughes, D.W. Eye tracking patterns in schizophrenia. Science 1973, 181: 179-181.

30. Latham, C., Holtzman, P.S., Manschreck, T.C. \& Tole, J. Optokinetic nystagmus and pursuit eye movements in schizophrenia. Arch Gen Psychiatry 1981, 38: $997-1003$.

31. Solomon, C.M., Holzman, P.S., Levin, S. \& Gale, H.J. The association between eye-tracking dysfunctions and thought disorder in psychosis. Arch Gen Psychiatry 1987, 44: 31-35.

32. Kinney, D.K., Woods, B.T. \& Yurgelun-Todd, D. Neurologic abnormalities in schizophrenic patients and their families: 11. Neurologic and psychiatric findings in relatives. Arch Gen Psychiatry 1986, 43: 665-668.

33. Francis, A. Validating schizotypal personality disorders: Problems with the schizophrenia connection. Schizophrenia Bulletin 1985, 11: 595-597.

34. Brinkley, J.R., Beitman, B.D. \& Friedel, R.O. Low dose neuroleptic regimes in the treatment of borderline patients. Arch Gen Psychiatry 1979, 36: 319-326.

35. Leone, N.F. Response of borderline patients to laxapine and chlorpromazine. J Clin Psychiatry 1982, 43: 148150.

36. Serban, G. \& Siegel, S. Response of borderline and schizotypal patients to small doses of thiothixene and haloperidol. Am J Psychiatry 1984, 141: 1455-1458. 
37. Soloff, P.H., George, A., Nathan, R.S., Schultz, P.M., Ulrich, R.F. \& Perel, J.M. Progress in pharmacotherapy of borderline disorders. Arch Gen Psychiatry 1986, 43: 691-697.

38. Goldberg, S.C., Schulz, S.C., Schulz, P.M., Resnick, R.J., Hamer, R.M. \& Friedel, R.O., Borderline and schizotypal personality disorders treated with low-dose thiothixene vs placebo. Arch Gen Psychiatry 1986, 43: $680-686$.

39. Lewis, N.D.C. \& Piotrowski, Z.A. Classical diagnosis of manic-depressive psychoses. In Zubin, J. (ed) Depression. Grune and Stratton, New York, 1954, pp 25-38. 